\title{
EDUCATION
}

\section{Clinical Education: MLS Student Perceptions}

\author{
JEANNE M. ISABEL
}

\begin{abstract}
This research study examines the perspectives of medical laboratory science (MLS) students completing their clinical education. Experiential learning provides the conceptual framework for clinical education in the healthcare professions. Students are placed into a community of practice where they are asked to apply learned theories in a sometimes stressful and distracting setting. A series of interviews and observations took place involving MLS students completing clinical internships. Participants were able to describe factors that contribute to the process of how learning occurs during clinical education. The four categories of findings that are described in depth include student self-awareness, the clinical environment and setting, preparation for clinical education, and preceptor role. Participants described the process of learning as one in which they apply skills learned prior to the internship with the experience of professional socialization, teamwork, and unique patient or interprofessional communication during the practicum. The role of preceptors is a key factor in providing an environment conducive to student learning. Conclusions and recommendations from these data provide valuable insight to educators of medical laboratory science and other healthcare professions in regards to clinical education.
\end{abstract}

ABBREVIATIONS: NAACLS - National Accrediting Agency for Clinical Laboratory Sciences, MLS Medical Laboratory Science

INDEX TERMS: Medical Laboratory Science, Clinical Education, Allied Health

Clin Lab Sci 2016;29(2):66-71

Jeanne M. Isabel, Ed.D, MLS,SH(ASCP), Medical Laboratory Science Northern Illinois University, DeKalb, $I L$
Address for Correspondence: Jeanne M. Isabel, Ed.D, $M L S, S H(A S C P), M L S$ Program Director and Associate Professor, Northern Illinois University, DeKalb, IL, 60115, 815-753-6330, jisabel@niu.edu

\section{INTRODUCTION}

Recruitment of MLS students for baccalaureate degree programs is critical to not only the survival of the profession but to the health and well-being of the general public. According to a report by the National Accrediting Agency for Clinical Laboratory Science (NAACLS), achievement of the necessary number of new graduates is becoming more challenging because the number of accredited MLS programs has dropped from 709 in 1975 to 224 in $2015 .{ }^{1}$ A key component of the MLS curriculum is clinical education known as a practicum or internship in a clinical setting. Because there is a need for MLS in the workforce, programs face the challenge of how to solicit support from clinical facilities to engage in clinical education. Finding placement for university based MLS program students in hospital laboratories may be affected by decreased laboratory staffing and technology upgrades. Other disciplines in the healthcare field have similar concerns. Academic healthcare programs are depending on clinical facilities to find a way to incorporate education into the busy workload. Best emphasizes that the demand for qualified laboratory professionals is likely to continue to increase and, by hosting students for clinical internships, hospitals have the opportunity to hire employees who can grow and develop within their organizations. ${ }^{2}$

\section{Study Purpose and Research Questions}

The purpose of this qualitative study was to examine the process of learning by MLS students through their lived experiences of clinical education. A total of six participants completing practicum internships were interviewed at two separate times. The participants were from four different universities and were placed in both large and small hospital laboratories. Participant descriptions of how learning occurs during clinical education provided ideas and strategies for preparing 


\section{EDUCATION}

future MLS students for professional practice. Participants also provided their perspectives on personal attributes and the clinical environment as a means of building confidence and competence in laboratory procedures during the learning process. Participants emphasized the importance of unique learning experiences and interprofessional interaction that occurred in the workplace environment.

In addition to participant interviews, the researcher's direct observation of eight different students in two large hospital laboratories during the education process provided additional evidence for analysis of student interaction with clinical instructors. By understanding MLS student learning in clinical education, new strategies for the process of preparing students for their role as professional practitioner can be practiced.

Participants described personal attributes, attitudes, knowledge and skills, the workplace environment, and the connections made between the classroom and "real life" setting. Through their own words, participants provided examples of how theory, practice, the environment, and professional socialization contributed to their learning experience to answer the following research questions.

$>$ According to participants, what personal attributes, knowledge, and skills contribute to learning during clinical education?

$>$ According to participants, what role does the workplace environment and clinical preceptor play in the learning process of clinical education?

$>$ How do participants describe preparation for clinical education and transfer-of-learning from the classroom and student laboratory to the hospital laboratory?

\section{MATERIALS AND METHODS}

Participants were recruited from NAACLS accredited MLS programs in a Midwestern state with the help of MLS Program Directors and clinical representatives. Communication with the volunteer participants took place through email or cell phone contact. A total of 6 MLS students, two males and four females, were interviewed face to face. Two separate interviews were conducted with 5 of the participants using a different interview guide for each. Comments from the first interview were incorporated into the second interview. Interviews were recorded for later transcription and took place either in the hospital setting or in an agreed upon location. Observation of eight students who were not interviewed provided insight to student/ instructor interaction in different departments of the laboratory. Field notes from the observations were also transcribed following the observations. In addition to interviews and field observations, participants were asked to submit a reflection summary of his/her experience during clinical education. A guide for the reflection paper was provided to the participant at the first interview and participants emailed the document to the researcher. The software program QSR NVivo10 was used to code all documents. ${ }^{3}$ In addition, the NVivo 10 program was used to query and explore for patterns and themes for analysis of findings.

\section{Findings}

This study was focused on participant descriptions of their learning process during clinical education and was not linked to student outcomes. Perceived success by participants was related through descriptions of how their learning occurred and what aspects of the learning environment were important. Although participants were in a variety of laboratory settings, their descriptions of the learning process were quite similar. The interpretation of how learning occurs in the clinical laboratory was facilitated by analysis of the similarities and differences described by participants and observed by the researcher. These findings provide relevance to the process of clinical education, which is a step in the preparation of students for professional practice within the hospital organization.

A table of findings (Table 1) was prepared to summarize topics and subtopics that emerged. A system of open coding was used to capture the meaning of what had been described by participants. The coding method as described by Saldana for the first cycle included both a holistic and exploratory approach to discover broad topics to align with the research questions. ${ }^{4}$ The second round of coding was a more structural process to split the broad topics into more descriptive phrases according to the narrative presented by participants with subsequent coding cycles done to answer the research questions posed. By looking at both similar and contrasting phrases from the participant's perspective, the researcher was able to identify themes on the process of learning as it occurs 
in clinical education.

\begin{tabular}{l}
\hline Table 1. Summary of Category Findings \\
\hline Categories \\
Student self-awareness facilitates learning through \\
- $\quad$ knowledge of strengths in laboratory skills and critical \\
thinking \\
- $\quad$ recognition of areas for improvement \\
knowledge of how learning occurs and how learning \\
styles can be recognized
\end{tabular}

Factors of the clinical environment and physical setting that affect learning include

- heavy workload that disrupts the ability of the student to ask questions

- unique interactions with patients such as phlebotomy

Student preparation for clinical education can depend on

- how the student organizes materials from preclinical learning

- types of relationships with instructors

- student understanding of methods of evaluation

The preceptor has an effect on student learning

- through socialization that helps build student confidence

- through demonstration of professionalism and teamwork as a mentor

- by demonstrating effective communication with other professionals

How students can discover MLS as a profession is linked to

- visibility of the profession

- good program information on websites

- evidence of career success in healthcare serving the community

\section{Student Self-Awareness}

All participants were able to identify individual factors they recognized as being helpful to the process of learning. Individuals reported on personal attribute strengths, laboratory skills learned at a previous job or during preclinical education and theoretical knowledge from didactic courses as means to facilitate learning during clinical education. One participant reported on success during the practicum experience in a reflection paper by stating the following, "As an MLS, I believe that I am a hard worker and a fast learner. I have been successful in my rotations."

Some of the personal attributes identified as contributing to learning success in clinical education included being observant, organized, detail-oriented, and able to communicate to a variety of audiences. MLS preclinical instructors often emphasize the need to pay attention to details when following procedures because of the importance of this trait in MLS practice.

Participants also described time management as an essential part of being organized. The need for creative scheduling to find time to read and study during the week when working essentially an eight-hour shift at the hospital for clinical practicum plus working a part-time job was described by each participant. At least two participants commented on the stress involved in commuting to different facilities that sometimes meant a long-distance travel from home.

Certain behaviors to facilitate clinical learning were described during interviews with participants. A common comment included the ability to exhibit an attitude of positivity and flexibility which can make an impression on practitioners in the clinical setting. One way in which this positivity can be applied to enhanced learning in clinical education is through coping strategies. Melincavage, who studied anxiety in nursing students to a degree that they might leave the education program, suggested that educators should strive for a better understanding of the anxiety and therefore develop interventions to decrease it. ${ }^{5}$ Participants described a level of stress or anxiety that contributed to their experiences by noting the difference between familiar and unfamiliar laboratory tests or equipment which often subsided with time and when confidence was developed. Through selfreflection on areas for improvement, participants often reported a need for additional help in a particular department of the clinical experience.

\section{How Learning Occurs}

Experiential learning, the basis for clinical education, relies on the interactions among student, environment, and instructor. ${ }^{6}$ Participants indicated that the way they showed interest and involvement in learning was by asking instructors questions through every step of the process. Variations between different departments of the laboratory were reported by the participants to be somewhat stressful at first until the routine was learned. The following table presents a model of the learning process in clinical education derived from participant descriptions. (Table 2). This model may be helpful to clinical instructors who have not previously worked with students in a clinical setting. 
Table 2. A Model of the Process of Clinical Learning

- Instructor demonstrates/student observes

- student practices/ Instructor observes

- student performs unobserved/Instructor checks results (Repeated as needed)

- Student works independently/ checks in with Instructor when needed

- Student gains confidence in performing tasks and is able to solve problems

\section{Physical Setting and Distractions}

The hospital setting as a learning environment for clinical education is a completely new experience for most students in healthcare programs. Only those who may have been a hospital employee or volunteer were aware of the typical atmosphere in this setting. Moving to an educational environment such as this may be stressful to students who have been accustomed to sitting in a classroom with peers. The hospital laboratories where observation took place were in an urban setting and the physical layout of each of these facilities consisted of one large room divided by partitions, modular furniture, and floor-model large instruments. Although it would seem that the noise level would be very high in such a large room, noise was not a major distracter. One of the greatest distractions was the movement of personnel from place to place while conversing with medical laboratory scientists or delivering specimens for testing.

Another key factor affecting learning during clinical education was the workload of the facility. One participant described the workload as "intense," which could be interpreted as "very busy." Another participant mentioned not being prepared for the atmosphere experienced in clinical education. Although workload is not something that can be predicted, it is clear that if a student must stand to the side during an emergency protocol, he or she is not able to ask questions. In this instance, the personal attribute of careful observation can be quite valuable. This scenario was described by more than one participant and they happily reported that after the emergency or event, the preceptor was able to explain what had taken place during the time when the student was on the sidelines. Through observation and social interaction with preceptors, students can gain a sense of comfort that can relieve some of the stress associated with learning the unfamiliar.

Although many aspects of the clinical environment can be unpredictable the opportunity to see unique learning events and practice more complex procedures that cannot be simulated in an academic setting are invaluable. Some of the scenarios of unique experiences described by participants were focused on the type of learning that occurred or a special case. Those participants that were introduced to phlebotomy also had interesting stories to tell related to patient interaction.

\section{Preparation for Clinical Education}

Although academic instructors try to prepare students for clinical education before they begin the experience, it is not unusual for students to state that they do not know what to expect. Many MLS programs ask students to visit hospital laboratories in preparation for clinical placement. That visit may be limited to a quick tour. Participants in this study responded that they either knew what to expect from previous laboratory work experience or thought the learning would be more of a "shadowing" experience with limited hands-on work.

Relationships with preclinical instructors and the ability to experience some laboratory procedures in a nonthreatening student lab was enough to help the interviewed participants feel prepared for some aspects of clinical education. There was a strong distinction between familiar and unfamiliar which had a lot to do with manual techniques versus automated equipment. Very few academic institutions are able to introduce students to a variety of automation prior to the clinical experience. According to participants of this study, successful learning during clinical education depends on the student's ability to be organized and focused during preclinical preparation and maintaining a positive attitude throughout the program.

\section{Role of Preceptor}

The relationships that developed between the participants and clinical instructors during the practicum had an impact on the level of learning and confidence experienced by the participants. Practitioners working with students need to be cognizant of the present-day diversity of students completing healthcare programs. 


\section{EDUCATION}

Many of the more experienced clinical instructors may have never attended a class on teaching methods or how to engage students in learning activities. These preceptors may not recognize the need for treating each student individually and teaching them according to personal attributes and learning styles. Although the process of teaching may be the same, successful learning by each student varies.

The nature of clinical education requires close interaction with professional practitioners who may also serve as coaches or cheerleaders. The role of the clinical instructor or preceptor is an important component of student success during clinical education learning. Since clinical learning often takes place with one on one instruction, it is the preceptor who monitors a student's progress, provides feedback, and serves as a general mentor for a student. There were some reported differences in participant preference for working side by side with more experienced practitioners or with recent graduates of MLS programs. Participants recognized the value associated with the wealth of knowledge a practitioner accumulates over time. Another participant was concerned that not all preceptors are given a choice of whether or not they are involved in teaching students which may have had an impact on the level of learning that transpires.

\section{DISCUSSION}

According to participants of this study, student success in MLS clinical education depends on self-awareness and the ability to identify one's strengths and skills. Being aware of how learning occurs and reflection on personal traits that assist with the learning process may lead to success in learning. Future studies would be beneficial to link actual student outcomes with student perceptions. Findings indicate that students with positive attitudes who show initiative and focus when in the learning environment make an impression on those who are teaching. Participants described an advantage to learning in clinical education included having experience working in a hospital or laboratory environment.

Participants who demonstrated understanding of critical thinking and its application in the community of practice called themselves successful learners. Participants reported that preparation of a reflective summary of the clinical experience was a valuable tool for recalling situations and events that made an impact. Chirema and
Boud suggest that journal writing as a reflective practice can help a student return to the lived experience when it has passed. ${ }^{78}$ Williams and Wessel also reported the value of student reflection on how learning occurs in order for the student to adopt coping strategies and become aware of individual roles. ${ }^{9}$

These findings indicate that when practitioners in the clinical environment are friendly and welcoming to all types of students, the adjustment to learning occurs rather quickly and successful learning results. Nickle describes the process of modeling, coaching, and mentoring by preceptors during clinical education of nurses. ${ }^{10}$ Preceptors for MLS students should also recognize the important role they play in the education of future laboratory practitioners.

Learning through observation is one way to prepare students for unusual and non-typical situations experienced during clinical education. This is also the opportunity for patient interaction. Students may be asked to step out of their comfort zones to address communication issues or other situations that require critical thinking and problem-solving. It is important to help students understand what variables occur in the clinical environment that are not controllable.

\section{Recommendations for MLS Students, Preceptors and} Academic Faculty

In order to be a successful learner during clinical education, MLS students should reflect on strengths recognized during preclinical education and learningstyle techniques that were most helpful. They should review basic skills mastered in preclinical education or previous laboratory experience and make logistic plans related to transportation and housing during the clinical practicum to relieve financial stress. Students must communicate with instructors and embrace new situations and learning opportunities that arise during clinical education. Finally, students need to prepare for clinical education by reviewing preclinical materials and making sure that methods of clinical evaluation are understood.

Conclusions from this study indicate the role of clinical preceptor to be an extremely important factor of MLS student learning during clinical education. One recommendation for preceptors includes taking advantage of continuing education opportunities related 
to teaching diverse student populations and informing their supervisors of the amount of time dedicated to being a preceptor. Instructors should also be friendly and welcoming, keeping in mind that most MLS students are not familiar with the clinical environment and may be intimidated by people or instruments. Preceptors should recognize that asking questions is a student's way of demonstrating focused learning on a topic and frequent feedback on his/her performance helps the student evaluate progress. Preceptors performing a difficult task should speak their thoughts out loud so that students can follow their critical-thinking pattern which is one of the best ways to transfer a process to a student. And last but not least, the preceptor should be a role model for professionalism, teamwork and communication with other healthcare professionals.

Recommendations for MLS program academic faculty who prepare students for learning in clinical education include following up on gaps of learning reported by students as they reflect on the transfer of learning from the university to the clinical laboratory. Faculty should also maintain positive relationships with clinical staff which may include preparation of an instructor manual or orientation session to guide inexperienced preceptors. Academic institutions may be able to offer continuing education events for preceptors and should give students preparing for clinical education detailed descriptions of the clinical environment and the assessments that will take place. Practicing communication skills during preclinical courses through role-playing and situational simulations is one way to guide students in strategies for coping with change and stress of a clinical environment. Helping students discover the profession of medical laboratory science through informative academic program websites with reports of positive outcomes is one way to promote the profession.

\section{Further Research}

Data collected from this study provides evidence important to the profession of medical laboratory science by showing how clinical education contributes to the goals of accreditation standards and expectations of the profession. Recommendations for further research in this area include linking student success outcomes such as certification board exam scores and clinical evaluation to student perceptions of success. An additional project for future research would be to develop an assessment tool to measure student success in clinical learning. Investigation of the process in which teaching laboratories select preceptors and how they can receive better recognition of their efforts would also be a valuable research study.

\section{REFERENCES}

1. Simonian Y. NAACLS update. Paper session presented at the CLEC meeting of the American Society of Clinical Laboratory Science, Cincinnati, OH. 2015 Feb.

2. Best ML. Avoiding crisis: Right-sizing staffing for the future. Clin Leader \& Mgmt Rev 2002;16(6):428-32.

3. QSR International Pty Ltd. NVivo(10) [software]. Victoria, Australia: Author. 2014.

4. Saldana J. The coding manual for qualitative researchers. 2nd ed. Thousand Oaks, CA: Sage; 2013.

5. Melincavage SM. Student nurses' experiences of anxiety in the clinical setting. Nurse Educ Today 2011;31(8):785-9.

6. Kolb DA. Experiential learning. Engelwood Cliffs, NJ: PrenticeHall, Inc; 1984.

7. Chirema KD. The use of reflective journals in the promotion of reflection and learning in post-registration nursing students. Nurse Ed Today 2007;27(3):192-202.

8. Boud D. Using journal writing to enhance reflective practice. New Dir for Adult and Cont Ed 2001;90:9-17.

9. Williams RM, \& Wessel J. Reflective journal writing to obtain student feedback about their learning during the study of chronic musculoskeletal conditions. J Allied Health 2004; 33(1):17-23.

10. Nickle P. Cognitive apprenticeship: Laying the groundwork for mentoring registered nurses in the intensive care unit. Dynamics 2007;18(4):19-27. 